\title{
Ureteral access sheath use in retrograde intrarenal surgery
}

\author{
Mustafa Karaaslan, Senol Tonyali, Mehmet Yilmaz, Sedat Yahsi, Sedat Tastemur, Erkan Olcucuoglu \\ University of Health Sciences, Turkiye Yuksek Ihtisas Training and Research Hospital, Clinic of Urology, Ankara.
}

\begin{abstract}
Summary Objective: To determine if there is a difference between postoperative urinary infection rates after retrograde intra-renal surgery (RIRS) when ureteral access sheath (UAS) was used or not used.

Materials and methods: We retrospectively analyzed the medical records of all patients who underwent RIRS at our institution between January 2016 and October 2018.

Results: 129 patients were included in the study. The mean age of the patients was $48.8 \pm 12.1$ years; 94 patients were male and 35 were female. The mean stone size (largest diameter), stone attenuation and stone volume were $15.3 \pm 5.8 \mathrm{~mm}, 1038$ $\pm 368 \mathrm{HU}$ and $1098 \pm 1031 \mathrm{~mm}^{3}$, respectively. Out of 129 patients, 81 were treated by using UAS (Group 1) and 48 were treated without use of UAS (Group 2). There was no statistically significant difference between the two groups in terms of post-operative infection $(p=0.608)$. However, the operative time of patients with post-operative infection was statistically higher than the other patients; $88.35 \pm 22.5$ min versus 59.37 $\pm 22.1 \mathrm{~min}(p=0.017)$. In multivariate regression analysis, operation time ( $p=0.02, r=1.07)$ was found to be the sole predictor of post-operative infection.

Conclusions: Using UAS during RIRS might reduce the intrarenal pressure and also has several advantages. However not prolonging the operation time too much could be of higher importance than UAS use in terms of preventing post-operative infection after RIRS.
\end{abstract}

KEY WORDS: Nephrolithiasis; Sepsis; Ureteral access sheath; Intrarenal pressure.

Submitted 27 February 2019; Accepted 21 March 2019

\section{INTRODUCTION}

Nephrolithiasis was historically treated with open surgery, however currently, minimally invasive modalities such as extracorporeal shoch wave lithotripsy (SWL), percutaneous nephrolithotripsy (PNL) and retrograde endoscopic interventions [ureteroscopy (URS), retrograde intrarenal surgery (RIRS)] and laparoscopic surgeries are frequently being the treatment of choice. RIRS is used more frequently by technological advancements of flexible ureterorenoscopes, which was first used by Marshall (1) in 1964 (2). PNL is recommended as the first-line treatment for kidney stones larger than $2 \mathrm{~cm}$ in the European Urology Association (EAU) guidelines. SWL or endoscopic procedures are recommended for renal stones smaller than $2 \mathrm{~cm}$. Flexible URS (fURS) could be presented as a second treatment option for stones larger than $2 \mathrm{~cm}$ with PNL being the first treatment option, and for lower pole stones larger than $1.5 \mathrm{~cm}$ where SWL activity was limited (3). When we look at the history of the use of ureteral access sheath (UAS) in fURS, for the first time Takayasu and Aso (4) used a Teflon tube throughout the ureter in 1974. The use of UAS in fURS has many advantages and disadvantages. UAS allows repeated access to the upper urinary tract, provides better visibility, allows removing small stone fragments without damaging the ureter, and reduces pyelolymphatic and pyelovenous reflux by lowering renal pelvic pressure (5). In addition, it was reported that it was not uncommon to have severe ureter wall damage during UAS placement into the ureter (6). In a multi-centre prospective study, it has been shown that using UAS in fURS might result in a decrease in the incidence of post-operative fever, urinary tract infection, and in particular sepsis (7).

In this study, we aimed to determine if there is a difference between postoperative urinary infection rates after fURS which UAS was used or was not used.

\section{MATERIALS AND METHOdS}

After obtaining the approval of institutional review board, we retrospectively analysed the medical records of all patients who underwent RIRS at our institution between January 2016 and October 2018. The study was conducted in accordance with the latest version of the Declaration of Helsinki. Informed consent form is not required due to the retrospective nature of the study.

Patients with anatomical abnormalities such as horseshoe kidney, pelvic kidneys, kidneys with multiple collecting system, and patients who underwent surgery under antibiotic supression were excluded from the study. All patients were evaluated with kidney-ureter-bladder (KUB) radiography, non-contrast abdominal computed tomography (CT), complete blood count, serum creatinine, bleeding and clotting times, complete urinalysis and urine culture. Patients who have bacteria growth in urine culture were treated with adequate antibiotic therapy and control urine culture confirmed no bacterial growth. The longest diameter of renal stone was determined as stone size.

The examined parameters included patient's demographic information, stone characteristics (size, volume, localization and Hounsfield unit), duration of operation, use of ureteral access sheath (UAS), stone-free rate, postoperative sepsis and urinary tract infection. Stone volume was calculated using the formula: stone volume = length $\mathrm{x}$ width $\mathrm{x}$ height $\mathrm{x} \pi \mathrm{x} 0.167$. The stone localization was defined as lower pole and non-lower pole. The diagnosis of sepsis was made by determining the focus of the infection and by 
the presence of two or more SIRS findings. The diagnosis of SIRS was based on the criteria of the American College of Chest Physicians/Society of Critical Care Medicine (ACCP/SCCM) Consensus Conference Committee as having 2 or more of the following criteria: 1 . body temperature less than $36^{\circ} \mathrm{C}$ or greater than $38^{\circ} \mathrm{C} ; 2$. heart rate greater than 90 beats $/ \mathrm{min} ; 3$. respiration rate $>20 /$ min or $\mathrm{PaCO}_{2}<32$ mmHg; 4. white blood cell counts greater than $12.000 / \mathrm{mm}^{3}$ or less than $4.000 / \mathrm{mm}^{3}(8)$.

\section{Surgical procedure}

All procedures were performed under general anesthesia by giving $1 \mathrm{gr}$ cefazolin prophylaxy before the procedure and using flexible ureteroscopes 7.5 and 7.8 French (Flex$X 2^{T M}$ Flex Scope, Karl Storz, Germany and Semi-flex Scope ${ }^{T M}$, MaxiFlex, USA). Patients were placed in the lithotomy position. Mobile C-armed fluoroscopy was used in all procedures. A 0.035 or 0.038 inch guidewire was inserted into the pelvis with a rigid ureterorenoscope. Then, 12/14F 45 $\mathrm{cm}$ (Rocamed) UAS was placed into the ureter till the ureteropelvic junction (UPJ) under the guidence of fluoroscopy. Alternatively, depending on the surgeon's preference, the operation was performed by reaching the kidney with flexible ureterorenoscope over the guidewire without inserting the UAS. Continuous irrigation was performed through fURS with $3000 \mathrm{~mL} \mathrm{0.3 \%} \mathrm{isotonic} \mathrm{solution} \mathrm{at} 50$ $\mathrm{cm}$ above the patient. A 270 micron holmium YAG laser was used for stone fragmentation. The stone fragments were left for spontaneous passage and no basket was used for stone removal.

\section{Statistical analysis}

Statistical analysis was performed using IBM SPSS Statistical Package v.22.0 for Windows (IBM SPSS Corp., Armonk, NY, USA). Quantitative values are shown as mean \pm standard deviation for parametric data and as median \pm range for nonparametric data. Qualitative values are shown in numbers and percentages. The normality test was performed using the Shapiro-Wilk test. The chi-square test was used to compare post-operative infection in patients with or without UAS, and Mann-Whitney U test was used to compare the duration of operation. Multivariate regression analysis was used to evaluate the most important determinant of post-operative infection. The level of statistical significance was accepted as $\mathrm{p}<0.05$.

\section{Results}

A total of 210 patients were reviewed. 129 patients who met the study criteria were included in the study.

The mean age of the patients was $48.8 \pm 12.1$ years; 94 patients were male and 35 were female. The mean stone size (largest diameter), stone attenuation and stone volume were $15.3 \pm 5.8 \mathrm{~mm}, 1038 \pm 368 \mathrm{HU}$ and $1098 \pm 1031$ $\mathrm{mm}^{3}$, respectively. Out of 129 patients, 81 were treated using UAS patients (Group 1) and 48 were treated without UAS (Group 2). The mean operative time was $60.2 \pm 22.8$ min. Stone free rate (SFR) was 52.7\% (68/129). Patient, stone and operation characteristics are given in Table 1. The mean stone size, stone volume, stone HU and operation time were similar in Group 1 and Group 2 ( $\mathrm{p}=0.34$, $\mathrm{p}=0.26$, p: 0.14 and p: 0.33, respectively). Lower pole stone localisation rate was significantly higher in Group 1 ( $62 \%$ versus $33 \%, p=0.002$ ). There was no statistically significant difference between the two groups in terms of post-operative infection $(p=0,608)$. However, the operative time of patients with post-operative infection was statistically higher than the other patients; $88.35 \pm 22.5 \mathrm{~min}$ versus $59.37 \pm 22.1 \mathrm{~min}(\mathrm{p}=0.017)$. In multivariate regression analysis operation time $(\mathrm{p}=0.02, \mathrm{r}=1.07)$ was found to be the sole predictor of post-operative infection whereas age, sex, stone volume, HU, stone location and UAS use were not, $(p=0.65, p=0.20, p=0.22, p=0.95$, $\mathrm{p}=0.35$ and $\mathrm{p}=0.78$, respectively).

\section{Discussion}

In 1964 , a stone in the ureter was observed by a $26 \mathrm{~F}$ cystoscope and the first fURS use was reported. By the end of the 1980s, the development of fURS has gained momentum. In the 1980s and 1990s initial fURS series were published (2). The new generation fURS allows the management of proximal ureteral and intrarenal pathologies, including complete removal of the ureter and kidney stones, with high success rates. Pyelovenous and pyelolymphatic reflux secondary to irrigation is one of the most important limitations of fURS (5).

Flexible URS is the first treatment option in many cases of treatment of kidney stones. It is recommended as the first treatment option in lower calyceal stones, especially between $1.5-2 \mathrm{~cm}$ (3). UASs were initially developed to facilitate difficult ureteroscopic access (9). Studies have shown the advantages and disadvantages of using UAS. The use of UAS has been shown to provide better view, multiple entries, removal of fragmented stones and, in particular, to reduce intrapelvic pressure (10). The results of studies on the effect of UAS use on SFR after RIRS differ. In a study conducted by Berquet et al. (11) on 280 patients, there was no difference in the SFR between the patients who were treated using UAS or not. Also in the present study SFR was similar in the two groups. There are UASs of different diameters and lengths. In the study by Wright et al. conducted with 10/12F and 12/14F UASs in cadaveric pig kidney, the mean intrapelvic pressure was $<40 \mathrm{~cm} \mathrm{H}_{2} \mathrm{O}$. When hand-assisted manual pump 
was used, it was shown that intrapelvic pressure increased up to $129 \mathrm{~cm} \mathrm{H}_{2} \mathrm{O}$ in cases treated with 10/12F UAS, and that this remained at low levels when $12 / 14 \mathrm{~F}$ UAS was used (12). In another study, intrarenal pressure during fURS was measured using a pressure transducer inserted from percutaneous nephrostomy in patients who had percutaneous nephrostomy due to obstructive ureteric stones. The mean pressure in the collecting system was $13.6 \mathrm{mmHg}\left(18.4 \mathrm{~cm} \mathrm{H}_{2} \mathrm{O}\right)$ before the procedure, while the mean intrarenal pressure in the renal pelvis during fURS rose to $94.4 \mathrm{mmHg}\left(128.3 \mathrm{~cm} \mathrm{H}_{2} \mathrm{O}\right)$ in patients without UAS and to $40.6 \mathrm{mmHg}\left(55 \mathrm{~cm} \mathrm{H}_{2} \mathrm{O}\right)$ in patients with UAS (5).

In a study conducted using human cadaveric kidney with continuous irrigation with a pressure of $200 \mathrm{~cm} \mathrm{H}_{2} \mathrm{O}$, renal pelvic pressure increased to $59 \mathrm{~cm} \mathrm{H}_{2} \mathrm{O}$ in patients without UAS, and intrapelvic pressure was reported to be below $30 \mathrm{~cm} \mathrm{H}_{2} \mathrm{O}$ in patients with UAS depending on the diameter and length of the UAS (13). In the present study, we used 12F/14F UAS in all cases and we did not use manual irrigation pump. We were not able to measure intrarenal pressure in renal pelvis, but, in the light of the aforementioned studies, our clinical practice might be associated with lower intrarenal pressures.

In a prospective study conducted with 2239 patients, it was shown that there was a decrease in the incidence of post-operative fever, urinary tract infection, and especially sepsis in patients who were treated by using UAS compared to patients who were treated without UAS (7). In a systematic review and meta-analysis, the efficacy and safety of UAS was evaluated in a total of 3099 patients, and SFR, intra-operative complications, duration of operation and length of hospital stay were found similar between UAS group and non-UAS group. Postoperative complications (bleeding, fever, urinary tract infection, bladder cramps, pulmonary embolism, and sepsis) were higher in patients treated by using UAS (14). In our study, no significant difference was found between patients who were treated with UAS or not in terms of post-operative infection. In-vitro studies have shown that pyelovenous reflux exists in case of a pelvic pressure $>35 \mathrm{mmHg}$ (15). The use of manual water pumps raises pressures even higher (12). This may increase the risk of postoperative infection by increasing pyelovenous reflux in parallel with the increase in intrapelvic pressure. In our study, high intrapelvic pressures should have been avoided because we did not use a manual pump so explaining the absence of a significant difference in postoperative infection between the group treated with UAS and the group treated without UAS.

In a study by Zhong et al., post-operative SIRS was detected in 21 (8.1\%) patients amomg 260 cases of fURS using UAS. The duration of operation was not statistically significant, but it was found to be higher in the SIRS group (16). In our present study, the operation time of the patients who developed post-operative infection was significantly higher than that of the other patients.

\section{Conclusions}

Using UAS during RIRS reduces the intrarenal pressure and has also several advantages. However not prolonging the operation time too much might be of greater impor- tance than the use of UAS in terms of preventing postoperative infection after RIRS.

\section{REFERENCES}

1. Marshall VF. Fiber optics in urology. J Urol. 1964; 91:110-114.

2. Van Cleynenbreugel B, Kilic O, Akand M. Retrograde intrarenal surgery for renal stones - Part 1. Turk J Urol. 2017; 43:112-121.

3. Türk C, Petfik A, Sarica K, et al. EAU guidelines on interventional treatment for urolithiasis. Eur Urol. 2016; 69:475-482.

4. Takayasu H, Aso Y. Recent development for pyeloureteroscopy: guide tube method for its introduction into the ureter. J Urol. 1974; 112:176-178.

5. Auge BK, Pietrow PK, Lallas CD, et al. Ureteral access sheath provides protection against elevated renal pressures during routine flexible ureteroscopic stone manipulation. J Endourol. 2004; 18:33-36.

6. Traxer O, Thomas A. Prospective evaluation and classification of ureteral wall injuries resulting from insertion of a ureteral access sheath during retrograde intrarenal surgery. J Urol. 2013; 189:580-584.

7. Traxer O, Wendt-Nordahl G, Sodha $H$, et al. Differences in renal stone treatment and outcomes for patients treated either with or without the support of a ureteral access sheath: The Clinical Research Office of the Endourological Society Ureteroscopy Global Study. World J Urol. 2015; 33:2137-2144.

8. Bone RC, Balk RA, Cerra FB, et al. Definitions for sepsis and organ failure and guidelines for the use of innovative therapies in sepsis. The ACCP/SCCM Consensus Conference Committee. American College of Chest Physicians/Society of Critical Care Medicine. Chest. 1992; 101:1644-1655.

9. Kourambas J, Byrne RR, Preminger GM. Dose a ureteral access sheath facilitate ureteroscopy? J Urol. 2001; 165:789-793.

10. Breda A, Territo A, López-Martínez JM. Benefits and risks of ureteral access sheaths for retrograde renal access. Cur Opin Urol. 2016; 26:70-75

11. Berquet $G$, Prunel P, Verhoest $G$, et al. The use of a ureteral access sheath does not improve stone-free rate after ureteroscopy for upper urinary tract stones. World J Urol. 2014; 32:229-232.

12. Wright A, Williams K, Somani B, Rukin N. Intrarenal pressure and irrigation flow with commonly used ureteric access sheaths and instruments. Central European J Urol. 2015; 68:434.

13. Rehman J, Monga M, Landman J, et al. Characterization of intrapelvic pressure during ureteropyeloscopy with ureteral access sheaths. Urology 2003; 61:713-718.

14. Huang J, Zhao Z, AlSmadi JK, et al. Use of the ureteral access sheath during ureteroscopy: A systematic review and meta-analysis. PloS one 2018; 13:e0193600.

15. Wang J, Zhou DQ, He M, et al. Effects of renal pelvic high-pressure perfusion on nephrons in a porcine pyonephrosis model. Exp Ther Med. 2013; 5:1389-1392.

16. Zhong W, Leto G, Wang L, Zeng G. Systemic inflammatory response syndrome after flexible ureteroscopic lithotripsy: a study of risk factors. J Endourol. 2015; 29: 25-8.

\author{
Correspondence \\ Mustafa Karaaslan - mustafakaraaslan23@gmail.com \\ Senol Tonyali, MD (Corresponding Author) \\ senoltonyali@hotmail.com \\ Mehmet Yilmaz - yilmazmehmet88@hotmail.com \\ Sedat Yahsi - sedatyahsi@yahoo.com \\ Sedat Tastemur - sedattastemur@yahoo.com \\ Erkan Olcucuoglu, MD - erkanolcucuoglu@gmail.com
}

